\title{
5-hydroxymethylcytosine but not MTAP methylation status can stratify malignant pleural mesothelioma based on the lineage of origin
}

Matteo Bosio ${ }^{1 \dagger}$, Elena Salvaterra ${ }^{1 \dagger}$, Francesca Datturi ${ }^{1 \dagger}$, Patrizia Morbini ${ }^{2}$, Michele Zorzetto ${ }^{1}$, Simona Inghilleri ${ }^{1}$, Stefano Tomaselli ${ }^{1}$, Patrizia Mangiarotti ${ }^{1}$, Federica Meloni ${ }^{1}$, Isa Cerveri ${ }^{1}$ and Giulia Maria Stella ${ }^{1 *}$

\begin{abstract}
Background: Malignant pleural mesothelioma (MPM) is an aggressive tumor with poor prognosis, mainly associated with work or environmental exposure to asbestos. MPM's molecular profile is largerly unexplored and effective therapies are still lacking. MPM rarely harbours those somatic genetic lesions that usually characterize solid epithelial-derived tumors. On this basis, our study aims at investigating MPM epigenetic profile.

Methods: We here assessed through immunohistochemistry, FISH and methylation specific PCR, the expression of 5-hydroxymethylcytosine (5- hmC) - an epigenetic marker and an important regulator of embryonic development and carcinogenesis - and the methylation status of the promoter of the MTAP gene - encoding for an enzyme involved in the rescue process of methionine and adenine - in two relevant series of FF-PE MPM samples derived from MPM thoracoscopic biopsies. Tissue sampling was performed at diagnosis.

Results: Within the limitations of the study cohort, the 5-hmC immunophenotype was different among the histological MPM types analysed. In fact, 18\% of the epithelial MPMs were negative, 47\% weakly positive, and 35\% of the cases showed an intense expression of 5-hmC. Sarcomatoid and biphasic MPMs showed intense 5-hmC expression pattern (positive and weakly positive in more than $80 \%$ of cases). Among MPM featuring epithelial lineage, none showed methylation of MTAP promoter.

Conclusions: Mesothelial sarcomatoid tumors featured a methylation profile characterized by a permanent gene silencing. Epithelial MPM methylation profile was in-between that of sarcomatoid MPM and the one of epithelial-derived tumors. MTAP promoter methylation level cannot be considered a suitable biomarker of epithelial MPM arousal.
\end{abstract}

Keywords: Malignant pleural mesothelioma, Cancer, Epigenetics, Methylation, Lineage of origin

\footnotetext{
* Correspondence: g.stella@smatteo.pv.it

${ }^{\dagger}$ Matteo Bosio, Elena Salvaterra and Francesca Datturi contributed equally to this work.

${ }^{1}$ IRCCS Fondazione Policlinico San Matteo- Unit of Respiratory System

Diseases, University of Pavia Medical School, Piazzale Golgi 19, 27100 Pavia,

Italy

Full list of author information is available at the end of the article
}

(c) The Author(s). 2018 Open Access This article is distributed under the terms of the Creative Commons Attribution 4.0 International License (http://creativecommons.org/licenses/by/4.0/), which permits unrestricted use, distribution, and reproduction in any medium, provided you give appropriate credit to the original author(s) and the source, provide a link to the Creative Commons license, and indicate if changes were made. The Creative Commons Public Domain Dedication waiver (http://creativecommons.org/publicdomain/zero/1.0/) applies to the data made available in this article, unless otherwise stated. 


\section{Background}

Malignant pleural mesothelioma (MPM) is an aggressive tumor which arises from pleural layer that is characterized by resistance to conventional treatment modalities and poor prognosis [1]. In the majority of cases, MPM is associated with work or environmental exposure to asbestos fibers $[1,2]$. Importantly, it can occur after a long latency [3]. The incidence of MPM is increasing and is expected to reach its peak by 2020 [4]. MPM's molecular profile is almost unknown so that the disease is still lacking effective therapeutic prospects. Recently, it has been shown that germline $B A P 1$ mutations are rare events that might predispose to MPM. Furthermore, somatic BAP1 changes are frequently reported [5], followed by mutations in $N F 2$ (encoding for merlin) and CDKN2A (encoding for $\mathrm{p} 16^{\mathrm{INK} 4 \mathrm{~A}}$ and $\left.\mathrm{p} 14^{\mathrm{ARF}}\right)$. Comprehensive genomic analysis allowed the identification of recurrent gene fusions and splice alteration as frequent mechanisms of inactivation of NF2, BAP1 in MPM and reported alterations in Hippo, mTOR, histone methylation RNA helicase and p53 signaling pathways [6]. Transcriptomic analysis demonstrated that poorest prognosis is associated to the activation of the epithelial-to-mesenchymal transition program which mainly affects sarcomatoid subtypes [7]. Recent insight in regarding epigenetic alterations in MPM showed that they are common events during disease onset and progression [8]. A better understanding of epigenetic mechanisms affecting MPM is, thus, mandatory to provide novel therapeutic opportunities against MPM.

On this basis, our study aimed at investigating in two relevant cohorts of MPM the expression of 5-hydroxymethylcytosine (5- hmC), an epigenetic marker and an important regulator of embryonic development and carcinogenesis [9]. Moreover, we investigated the methylation status of the promoter of the methylthioadenosine phosphorylase (MTAP) gene, encoding for an enzyme involved in the rescue process of methionine and adenine. Inactivation of this gene - which is known to be involved in oncogenesis of different malignancies - may occur through two different mechanisms: i) genetic deletion; ii) hypermethylation of the promoter. Many solid tumours and hematologic malignancies lack expression of the MTAP enzyme, due to either deletion of the MTAP gene or methylation of the MTAP promoter. Solid tumors frequently lacking MTAP include MPM, non-small cell lung cancer (NSCLC), gliomas and pancreatic cancer. The hypermethylation of MTAP promoter is also involved in hepatocellular carcinoma as well as gastric adenocarcinoma onset [10, 11]. MTAP is located at the INK4 locus near the tumour suppressor gene $\mathrm{p} 16^{\mathrm{INK} 4 \mathrm{~A}}$. Homozygous deletion of CDKN2A (p16) is one of the most common genetic alterations in pleural mesotheliomas, occurring in up to $74 \%$ of cases $[12,13]$. MTAP resides in the same gene cluster of the $9 \mathrm{p} 21$ region and is co-deleted in the majority of CDKN2A deleted cases (90\%) $[11,12,14]$. Within regard to MPM, it has been recently reported that MTAP is frequently deleted. The combination of MTAP and BPA1 expression levels, detected by immunohistochemistry, appears to be a reliable and useful method for differentiating MPM cell from reactive mesothelial cells [15] with a good sensitivity and 100\% specificity in detecting MPM [16].

\section{Methods}

\section{Cases identification and selection}

A total of forty formalin-fixed paraffin-embedded (FFPE) samples derived from thoracoscopic biopsies of MPM patients was consecutively retrieved from the archives of the Pathology Division of the IRCCS Fondazione Policlinico San Matteo Hospital. For each case, exhaustive clinical data were also available. The study received ethical approval from local institutional review boards. Out of the 40 cases, 10 were female $(25 \%)$ and $30(75 \%)$ were male; the mean age at diagnosis was $67,57 \pm 9,03$ years. Out of them - accordingly to pathologic diagnosis - 15 cases were sarcomatoid MPM, 7 biphasic types and the remaining 18 cases were epitheliod tumors. Six out of the 40 analyzed patients reported a proved work exposure to asbestos fibers; overall the vast majority of cases (95\%-38 patients) referred environmental exposure. All patients featured advanced disease and underwent conventional chemotherapy (platinum/ pemetrexed) as first line approach. The overall survival of the analysed cohort was 14.87 months (st: \pm 9.40$)$. Clinical data are listed in detail in Table 1.

A second cohort, represented by a series of ninety FFPE blocks from surgical biopsies epithelial MPM, was available as well.

In all cases, tissue sampling was performed at diagnosis (before the beginning of chemo- and radiotherapy).

\section{Cytogenetic analysis Immunohistochemistry (IHC)}

Immunohistochemical analysis as well as fluorescence in situ hybridization study have been performed in order to correlate protein expression and homozygous deletion in mesothelioma tissues. The immunohistochemical protocol for 5-mhC staining used in this report was performed according to the previously optimized and validated method by Haffner et al. [17]. The 5 -hmC staining intensity was scored as none (0), weak (1), moderate (2) or marked (3), according to Lian et al. [18]. In detail $0=$ no immunolabeling; + = less intense than immunolabeling of in adjacent benign cells; $++=$ comparable with normal nuclei; and $+++=$ more intense than normal nuclei.

Immunostaining for MTAP was performed according to Kinoshita et al. [14] and scored as follows: very strong expression $(+++)$, strong expression $(++)$ low level of expression $(+)$. As a control, we checked 
Table 1 Clinical and demographic data of the analysed cohort

\begin{tabular}{|c|c|c|c|c|c|c|c|c|}
\hline & Patient ID & Gender & Age at diagnosis & Histology & Exposure to asbestos & TNM stage & Therapy & OS (months) \\
\hline 1 & M01 & M & 47 & $E$ & Work & IV & $S+C$ & 7 \\
\hline 2 & M02 & M & 77 & S & & IV & $S+C$ & 13 \\
\hline 3 & M03 & M & 70 & E & & IV & C & 43 \\
\hline 4 & M04 & M & 57 & S & Work & IV & C & 6 \\
\hline 5 & M05 & M & 70 & E & Work & IV & $S+C$ & 11 \\
\hline 6 & M06 & $\mathrm{F}$ & 70 & B & & IV & C & 8 \\
\hline 7 & M07 & M & 72 & S & & IV & C & 10 \\
\hline 8 & M08 & M & 64 & $E$ & & IV & $S+C$ & 5 \\
\hline 9 & M09 & $\mathrm{F}$ & 66 & $E$ & & IV & C & 18 \\
\hline 10 & M10 & $\mathrm{F}$ & 71 & $E$ & & IV & C & 4 \\
\hline 11 & M11 & M & 78 & S & & IV & $C+R$ & 13 \\
\hline 12 & $\mathrm{M} 12$ & M & 76 & $E$ & & IV & $C$ & 5 \\
\hline 13 & M13 & $\mathrm{F}$ & 70 & $E$ & Work & IV & C & 7 \\
\hline 14 & M14 & M & 72 & S & & IV & $C+R$ & 15 \\
\hline 15 & M15 & $\mathrm{F}$ & 77 & S & & $\| I \mathrm{~B}$ & $S+C$ & 21 \\
\hline 16 & M16 & $M$ & 75 & $E$ & & IV & $C$ & 9 \\
\hline 17 & M17 & $M$ & 70 & $S$ & & IV & $C$ & 15 \\
\hline 18 & M18 & $\mathrm{F}$ & 76 & $E$ & & $\| I B$ & $S+C$ & 24 \\
\hline 19 & M19 & M & 72 & B & & IV & $S+C$ & 7 \\
\hline 20 & M20 & $M$ & 80 & E & & IV & $S+C$ & 10 \\
\hline 21 & M21 & M & 74 & S & & IV & $S+C$ & 18 \\
\hline 22 & M22 & M & 60 & B & & IV & $S+C$ & 26 \\
\hline 23 & M26 & M & 57 & S & & IV & $S+C$ & 19 \\
\hline 24 & M29 & M & 64 & S & & IV & C & 19 \\
\hline 25 & M32 & M & 74 & $E$ & & IV & $C+R$ & 26 \\
\hline 26 & M33 & M & 71 & B & & IV & $S+C$ & 6 \\
\hline 27 & M34 & M & 75 & $E$ & & IV & $S+C$ & 26 \\
\hline 28 & M35 & $M$ & 60 & $S$ & & IV & $S+C$ & 47 \\
\hline 29 & M37 & M & 57 & $E$ & & IV & $S+C$ & 16 \\
\hline 30 & M41 & M & 44 & B & & IV & $S+C$ & 7 \\
\hline 31 & M42 & M & 60 & S & & IV & $S+C$ & 13 \\
\hline 32 & M45 & M & 73 & $E$ & & IV & $S+C$ & 12 \\
\hline 33 & M47 & M & 68 & $S$ & & IV & $S+C$ & 15 \\
\hline 34 & M48 & $\mathrm{F}$ & 58 & $E$ & & $\| I B$ & $S+C$ & 7 \\
\hline 35 & M77 & M & 56 & B & & IV & $C$ & 6 \\
\hline 36 & M78 & $\mathrm{F}$ & 80 & S & Work & IV & $S+C$ & 15 \\
\hline 37 & M79 & $\mathrm{F}$ & 72 & $E$ & & IV & $S+C$ & 19 \\
\hline 38 & M80 & M & 70 & B & & IV & $S+C$ & 20 \\
\hline 39 & M81 & M & 48 & S & & IV & $S+C$ & 16 \\
\hline 40 & M84 & $F$ & 63 & E & Work & IV & $S+C$ & 11 \\
\hline
\end{tabular}

E stands for epitheliod MPM, S: sarcomatoid, B: biphasic types; S stands for surgery, C for chemotherapy, R for radiotherapy

the expression of $5-\mathrm{hmC}$ and MTAP in a series of biopsies from reactive mesothelial hyperplasia ( $R M H)$ samples as well as from different proliferative lung pathologies: inflammatory conditions (idiopathic pulmonary fibrosis (IPF / UIP) and cryptogenic organizing pneumonia (COP), and cancer (adenocarcinoma 
(ADC), squamous-cell carcinoma (SCC)) and healthy lung tissue samples obtained from lobectomies performed to resect tumor masses.

\section{Methylation specific PCR and FISH analysis}

The method used to quantify promoter MTAP hyper methylation was sensitive melting analysis after real-time methylation specific-PCR (SMART-MSP), a diagnostic tool that permits to quantify the methylation levels of genes considered promising DNA methylation biomarkers for early cancer diagnostics. The methylation specific PCR has been conducted as already published [19]. The FISH was performed on the entire INK4 locus was performed on $4-\mu \mathrm{m}$-thick tissue cell block sections as previously described [14].

Details for both methods are described in the Supplementary Material Section.

\section{Statistical analysis}

Five-hmC scores were analyzed as interval data sets using two-sided Student's t-test. A $p \leq 0.05$ was considered statistically significant. Kappa statistics were used to assess the correlation between IHC expression of MTAP and homozygous deletion status of 9p21 FISH in cell blocks. All statistical analyses were performed using $\mathrm{R}$ statistical software (version 3.2.2; $\mathrm{R}$ Foundation for Statistical Computing, Vienna, Austria).

\section{Results}

\section{5-hmC expression}

Out of the 18 biopsies related to epithelioid MPM, 13 featured negative expression and in 5 cases an intense expression was revealed. The sarcomatoid histotypes and biphasic ones showed a more intense pattern of expression, higher than $50 \%$ of the cases of each group, compared to those with an epithelioid histotype (Table 2). Obviously, the small number of samples carrying sarcomatoid and biphasic histotype does not allow a more extensive conclusion; however, a clear prevalence of a more intense expression might be featured by tumors with mesenchymal lineage of origin.

As control, we considered the expression of 5-hmC in a series of RMH: none of them harbored positive staining. Moreover, we selected pulmonary biopsies of patients with idiopathic pulmonary fibrosis (IPF/UIP), cryptogenic pneumonia in organization (COP), adenocarcinoma (ADC), squamous cell carcinoma (SCC) and in healthy lung tissue samples. A general positivity at 5 -hmC was reported in samples of healthy lungs and COP. We documented both in the ADCs and in the SCCs observed a negative/weak marker expression. The $25 \%$ of the IPF/UIP samples showed a pattern like that of healthy controls and COP, characterized by a strong expression in the bronchial and alveolar epithelial cells. The remaining $75 \%$ of the fibrotic
Table 2 Results of 5-hmC immunohistochemistry analysis

\begin{tabular}{llll}
\hline 5-hmC IHC & & Positive & Negative \\
\hline MPM & E & 5 & 13 \\
& S & 9 & 6 \\
& B & 5 & 2 \\
RHM & & 6 & 19 \\
COP & ADC & 5 & 15 \\
NSCLC & SCC & 0 & 25 \\
& & 0 & 25 \\
IPF & 0 & 15 \\
Healthy lung & 0 & 25 \\
Sensitivity MPM/RMH = 47\% & & \\
Specificity MPM/RMH = 76\% & & \\
Sensitivity E/B $+\mathrm{S}=63 \%$ & & \\
Specificity E/B $+\mathrm{S}=72 \%$ & &
\end{tabular}

E stands for epitheliod MPM, S: sarcomatoid, B: biphasic types

samples showed a moderate/negative expression in the bronchiolar cells, in the cells of the terminal bronchioles and in the pneumocytes of type II activated (data not shown). Overall, the analysis documented a low sensitivity of the analysis (47\%) in distinguishing MPM and RMH whit an acceptable specificity (76\%). Within respect to MPM the test displayed a good sensitivity (63\%) and specificity (72\%) in stratifying cases based on the lineage of origin (Table 2).

\section{MTAP expression}

One hundred and thirty (130) samples were checked for MTAP expression by immunohistochemistry staining. The cohort derived from the previously described one (40 cases) and a series of ninety epithelioid MPM. Out of them, only two samples $(2,22 \%)$ were negative for MTAP expression. Some tumours contain more intensities as +/- indicating that samples showed different degrees of MTAP expression even though in some cases the staining was weak. Four main groups were defined. The first group consisted of samples that expressed MTAP protein very strongly $(+++)$, the second contained samples that expressed MTAP strongly $(++)$ and the third comprised samples that expressed MTAP at low level (Fig. 1.). Finally, samples showing a mix between cells expressing MTAP and cells lacking it, belonged to the fourth group $(+/-)$. To be assessed as MPM, the cells had to be positive for the two MPM markers: calretinin and KL1 [20]. We had available 89 fluorescence in situ hybridization results from MPM samples even if 6 out of them showed no data. Heterozygous deletion of the locus INK4 was not observed in any MTAP positive tumours. Sixty-four (64) samples showed no deletion confirming the IHC results, while 19 of them presented homozygous deletion. The results of the SMART-MSP 


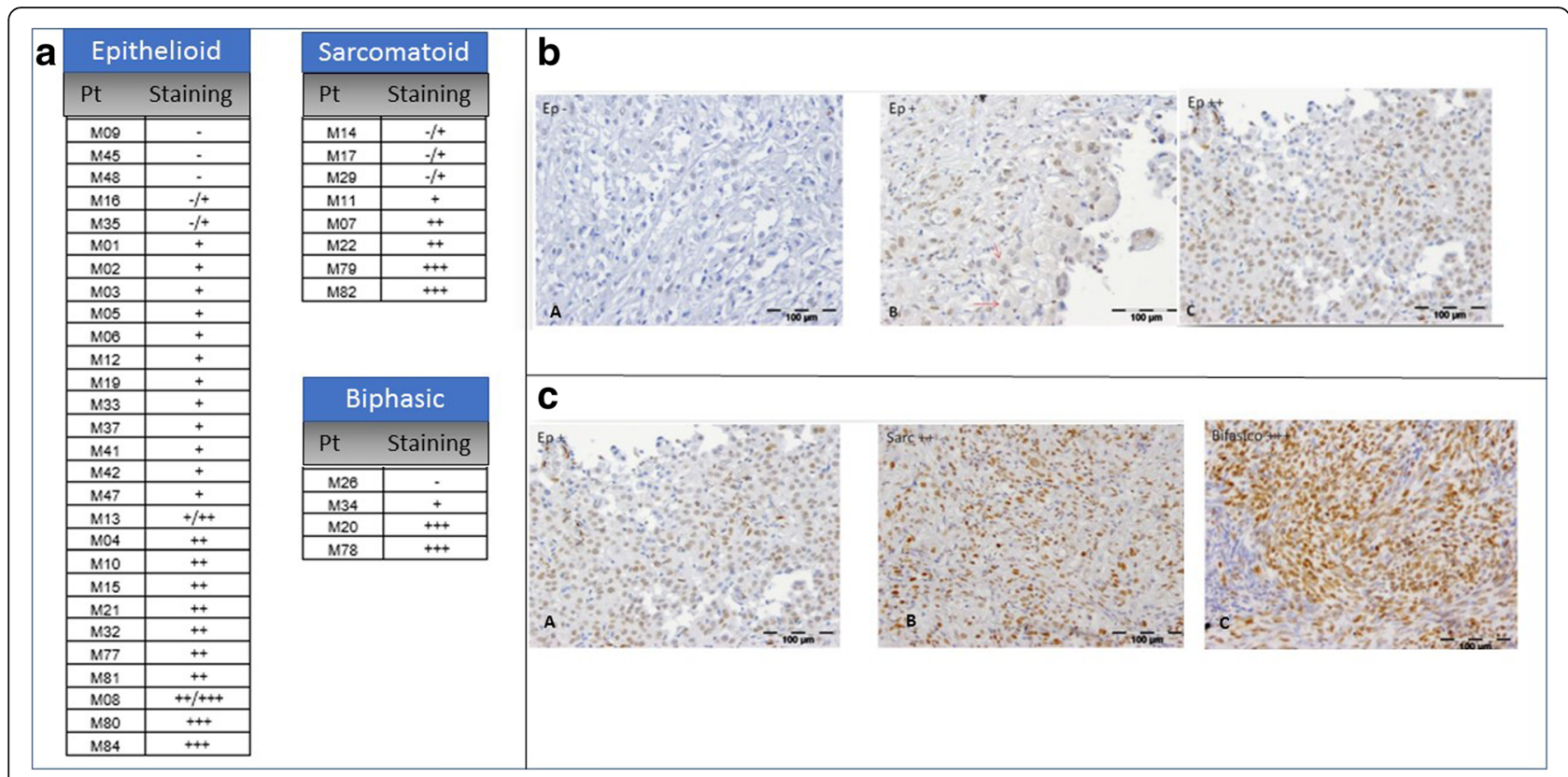

Fig. 1 Panel A. Immunohistochemistry staining for 5-mhC: semiquantitative score for each case and subtype. Panel B. Different levels of 5-mhC expression by $\mathrm{HC}$ among epitheliod mesothelioma samples. A: negative; B weakly positive; C: highly positive. Panel C. Different levels of 5-mhC expression by $\mathrm{IHC}$ among mesothelioma subtypes. A: weakly positive epitheliod MPM, B: intensely positive sarcomatoid MPM; C: strong intensely positive biphasic MPM type, featuring prevalence of sarcomatoid cells

assay revealed that no one of the samples analysed was methylated (Fig. 2).

What was difficult to explain is why there were 16 FISH results $(19,5 \%)$ that showed homozygous deletion of the locus INK4 even if the MTAP protein was expressed. One of the reasons could be due to experimental issues.
The FISH probe, in fact, was made of small complementary DNA fragments and it was not a large fragment that span the entire locus INK4. This led to the fact that, even the MTAP was not deleted but p16 was, the signal was too weak to be detected by the fluorescence microscopy. From the Real-time PCR we knew that there was a

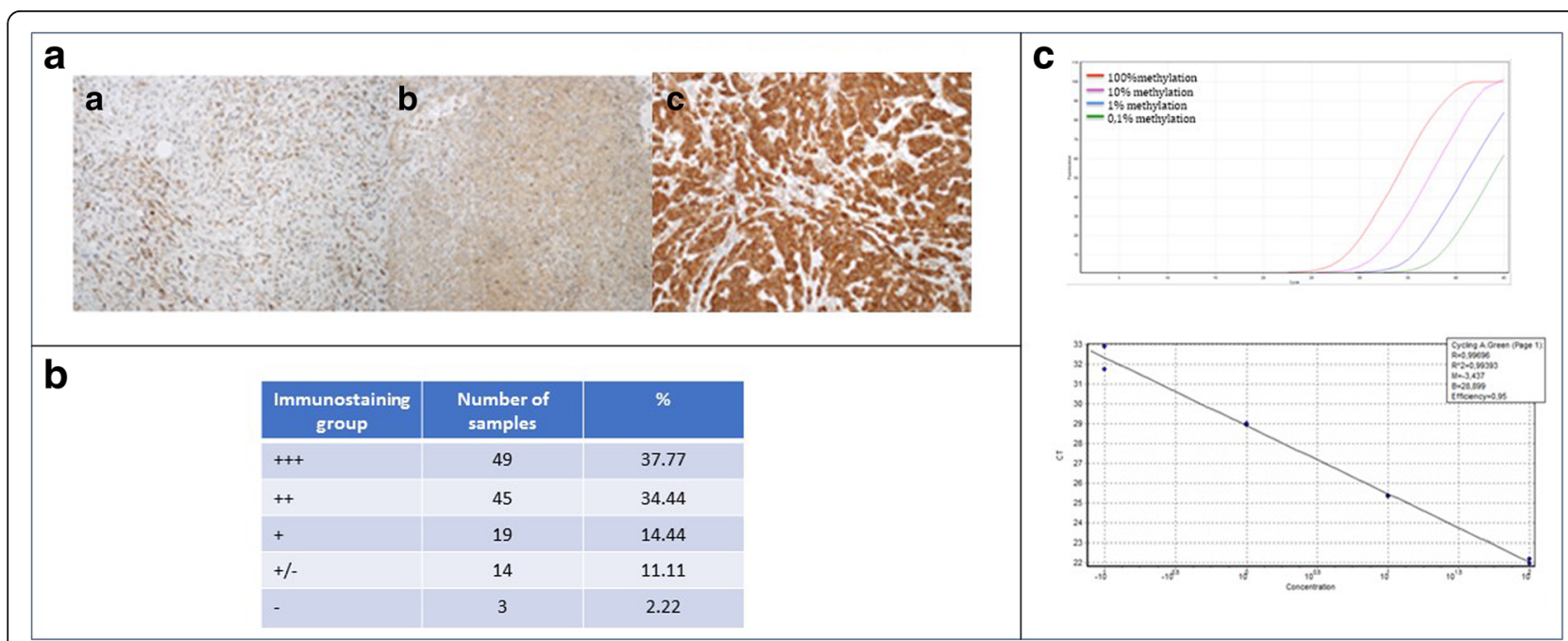

Fig. 2 Panel A. Immunohistochemistry staining for INK4 locus. A. INK4 locus immunohistochemistry staining of sample 4, group +/-. B. INK4 locus immunohistochemistry staining of sample 3, group ++. C. INK4 locus immunohistochemistry staining of sample 47 , group +++. Panel B. Immuno-staining groups and relative percentages ( $40+90$ cases analyzed). Panel C. Sensitivity and quantitative accuracy of MTAP SMART-MSP assay (90 cases analyzed). Top: The assay was sensitive to 0,1\% methylation in a background of WGA. Bottom: The assay was quantitative accurate in the range $100 \%$ methylation to $0,1 \%$ methylation ( $R 2=0,99,393)$. The PCR efficiency was 0,95 
template because the results showed that there was amplification of MTAP. Another possible explanation could be hemyzygous deletion not detected by the FISH analysis. A previous IHC study reported a complete loss of immunoreactivity in only $19 \%$ of the tumours, whereas $45 \%$ had some degree of retained protein expression in a cohort of 99 MPMs [21]. They hypothesized that some tumours may only harbour a hemizygous deletion rather than the homozygous deletion leading to complete protein loss. From these evidences we can assess that in the sequence in between the two genes MTAP and $p 16$ there is a breakpoint region more prone to be deleted. Investigating the database Gene (NCBI) we knew that this region could be LOC100533725 HERV-FRD provirus ancestral Env polyprotein pseudogene. Moreover, the use of dual-colour probes during the FISH analysis could give false positive results for deletions. We could not confirm the presence of false positive results for deletion in the FISH analysis, neither the existence of a breakpoint region between MTAP and $p 16$. Our results indicated that MTAP promoter hypermethylation seemed not to be involved in MPM carcinogenesis and that the MTAP and p16 co-deletion was present in none of the analysed 90 MPM samples. Overall, we could conclude that MTAP hypermethylation might not behave as a good biomarker for malignant mesothelioma.

\section{Discussion}

Although genomic alterations play a driving role, more recent evidence shows that changes that are not directly implicated in the DNA sequence also play an important role in cancer development. These epigenetic modifications affect temporal and spatial control of gene activity required for homeostasis of complex organisms. The global epigenetic profile determined by high-throughput methylation analysis differs between MPM and normal pleura, indicating that MPM, like other cancers, has aberrant CpG island methylation [7]. Globally these data suggested that the expression level of $5-\mathrm{hmC}$ is significantly reduced in human tumors and this is consistent with the complexity of the epigenomic alterations that characterize malignant proliferation. Furthermore, the depletion of 5-hmC - detected by immunohistochemical analysis - can constitute a biomarker usable in the diagnosis of cancer. It is well documented that in MPM - differently from solid tumors of epithelial origin - known somatic alterations activating oncogenes or inactivating tumor suppressors are rarely found $[5,22]$. It has been thus further investigated the epigenetic profile characterizing MPM. Even with the limits of the study cohort, immunohistochemical analyses aimed at documenting the level of 5-hmC expression showed conflicting results in different MPM subtypes with globally low expression in epithelioid forms versus higher levels in the sarcomatoid ones. Notably, results are not affected by MPM exposure to chemo agents and/or ionizing radiation. Therefore, it seems probable to conclude that the different lineage of origin can play a role in the methylation status of the different tumor subtypes. Thus, those tumors that originate from mesodermal derived cells (mesothelioma, mainly the sarcomatoid histotype) have an 'atypical' methylation profile characterized by elevated levels of $5-\mathrm{hmC}$. To deeper investigate these data, the MTAP expression was analyzed in a parallel cohort of 90 epithelioid MPM samples. The methylation status of the MTAP promoter was studied by designing a specific primer for the SMART-MSP assay, in order to quantify the methylation level of biopsy samples. Unexpectedly, none of the 90 samples of MPM analyzed shows methylation of the MTAP promoter. These results, although preliminary, suggested that hypermethylation of the MTAP promoter seems not to be involved in the onset of MPM featuring epithelial lineage. It can therefore be concluded that the latter cannot be considered a suitable biomarker to determine epithelial MPM onset and progression. An open problem remains linked to the need for defining the exact starting sequence of the deletion between MTAP and $p 16$. Overall, the results of this screening are consistent with the extreme biomolecular heterogeneity that characterizes MPM and explain the complexity of the approaches required for a more in-depth definition of the pathogenetic mechanisms and of targeted therapeutic options.

\section{Conclusions}

Overall, a prevalence of sarcomatoid mesotheliomas showed a methylation profile recalling what has been called 'epigenetic cancer stem cell signature, characterized by a permanent gene silencing, which favors the stay of the cell in one self-renewal state, predisposing it to malignant transformation [23]. In this global framework, the absence of MTAP promoter methylation is likely to be related to the fact that the gene is silenced primarily as a result of deletion, as already reported in the literature. The epitheliod mesotheliomas instead show a profile straddling the previous one and the one typical of epithelial-derived tumors, characterized by 5-hmC depletion. A second key point is linked to the need for a more in-depth characterization of the molecular mechanisms through which asbestos nanofibers might affect gene expression, even including methylation status by their direct interaction with chromatin.

\section{Abbreviations \\ 5-hmC: 5-hydroxymethylcytosine; ADC: Adenocarcinoma; COP: Cryptogenic organising pneumonia; FFPE: Formalin fixed paraffin embedded; \\ IPF: Idiopathic pulmonary fibrosis; MPM: Malignant pleural mesothelioma; MTAP: Methylthioadenosine phosphorylase; SCC: Squamous cell carcinoma}

\section{Acknowledgements}

The authors thank Andrea Marchelli for fruitful discussion and constructive comments and for critical reading of the manuscript. 


\section{Availability of data and materials}

The datasets generated and/or analysed during the current study are not publicly available since they are related to patient samples, but are available from the corresponding author on reasonable request at g.stella@smatteo.pv.it

\section{Authors' contributions}

$M B, E S, F D, M Z, S I, G S$ performed the experiments; GS FD, MZ designed the study, GM, PM, ST, FM, IC selected cases, GS, FD, MZ wrote the paper. All authors read and approved the final manuscript.

\section{Ethics approval and consent to participate}

The study received ethical approval from local institutional review boards for analyzing MPM samples.

\section{Consent for publication}

Not applicable.

\section{Competing interests}

The authors declare that they have no competing interests.

\section{Publisher's Note}

Springer Nature remains neutral with regard to jurisdictional claims in published maps and institutional affiliations.

\section{Author details}

${ }^{1}$ IRCCS Fondazione Policlinico San Matteo- Unit of Respiratory System Diseases, University of Pavia Medical School, Piazzale Golgi 19, 27100 Pavia, Italy. ${ }^{2}$ RCCS Fondazione Policlinico San Matteo- Pathology Unit, University of Pavia Medical School, Pavia, Italy.

Received: 10 March 2018 Accepted: 18 June 2018

Published online: 02 August 2018

\section{References}

1. Carbone M, Kratzke RA, Testa JR. The pathogenesis of mesothelioma. Semin Oncol. 2002;29(1):2-17.

2. Stella GM. Carbon nanotubes and pleural damage: perspectives of nanosafety in the light of asbestos experience. Biointerphases. 2011;6(2):P1-17.

3. Bianchi C, Giarelli L, Grandi G, Brollo A, Ramani L, Zuch C. Latency periods in asbestos-related mesothelioma of the pleura. Eur J Cancer Prev. 1997;6: 162-6.

4. Robinson BM. Malignant pleural mesothelioma: an epidemiological perspective. Ann Cardiothorac Surg. 2012;1(4):491-6.

5. Carbone M, Yang H, Pass HI, Krausz T, Testa JR, Gaudino G. BAP1 and cancer. Nat Rev Cancer. 2013:13(3):153-9.

6. Bueno R, Stawinski EW, Goldstein LD, Durinck S, De Rienzo A, Modrusan Z, et al. Comprehensive genomic analyss of malignant pleural mesothelioma identifiesrecurrent mutations, gene fusions and splicing alterations. Nat Genet. 2016:48(4):407-16.

7. De Reynies A, Jaurand MC, Renier A, Couchy G, Hysi I, Elarouci N, et al. Molecular classification of malignat pleural mesothelioma:identification of a poor prognosis subgroup linked to epithelila-to-mesenchymal transition. Clin Cancer Res. 2014;20(5):1323:34.

8. McLoughlin KC, Kaufan AS, Schrump DS. Targeting the epigenome in malignant pleural mesothelioma. Transl Lung Cancer Res. 2017;6(3):30-365.

9. Pfeifer GP, Kadam S, Jin SG. 5-hydroxymethylcytosine and its potential roles in development and cancer. Epigenetics Chromatin. 2013;6:10.

10. Hellerbrand C, Mühlbauer M, Wallner S, Schuierer M, Behrmann I, Bataille F, et al. Promoter-hypermethylation is causing functional relevant downregulation of methylthioadenosine phosphorylase (MTAP) expression in hepatocellular carcinoma. Carcinogenesis. 2006;27:64-72.

11. Leal M, Lima E, Silva P, Assumpção $P$, Calcagno D, Payão $S$, et al. Promoter hypermethylation of CDH1, FHIT, MTAP and PLAGL1 in gastric adenocarcinoma in individuals from northern Brazil. World J Gastroenterol. 2007;13:2568-74.

12. Ladanyi M. Implications of P16/CDKN2A deletion in pleural mesotheliomas. Lung Cancer. 2005:49:595-8.

13. Illei PB, Rusch WW, Zakowski MF, Ladanyi M. Homozygous deletion of CDKN2A and codeletion of the methylthioadenosine phosphorylase gene in the majority of pleural mesotheliomas. Clin Cancer Res. 2003;9:2108-13.
14. Kransiskas AM, Bartlett DL, Cieply K, Dacic S. CDKN2A and MTAP deletions in peritoneals mesotheliomas are correlated with loss of p16 protein expression and poor survival. Mod Pathol. 2010;23:531-8.

15. Kinoshita Y, Hida T, Hamasaki M, Matsumoto S, Sato A, Tsujimura T, et al. A combination of MTAP adn BAP1 immunohistchemistry in pleural effusion cytology for the diagnosis od mesothelioma. Cancer.Cytopathol. 2018; 126(1):54-63.

16. Hida T, Hamasaki M, Matsumoto S, Sato A, Tsujimura T, Kawahara K, et al. Immunohistochemical detection of MTAP and BAP1 protein loss for mesothelioma diagnosis: comparson with 9p21 FISH and BAP1 immunohistchemistry. Lung Cancer. 2017;104:98-105.

17. Haffner MC, Chaux A, Meeker AK, Esopi DM, Gerber J, Pellakuru LG, et al. Global 5-hydroxymethylcytosine content is significantly reduced in tissue stem/progenitor cell compartments and in human cancers. Oncotarget. 2011:2(8):627-37.

18. Lian CG, Xu Y, Ceol C, Wu F, Larson A, Dresser K, et al. Loss of 5hydroxymethylcytosine is an epigenetic hallmark of melanoma. Cell. 2012; 150(6):1135-46.

19. Herman JG, Graff JR, Myöhänen S, Nelkin BD, Baylin SB. Methylation-specific PCR: a novel PCR assay for methylation status of CpG islands. Proc Natl Acad Sci U S A. 1996;93(18):9821-6.

20. Robinson BW, Creaney J, Lake R, Nowak A, Musk AW, de Klerk N, et al. Mesothelin-family proteins and diagnosis of mesothelioma. Lancet. 2003; 362:1612-161.

21. Zimling ZG, Jorgesen A, Santoni-Rugiu E. The diagnostic value of immunohistochemically detected methyoadenosine phoshorylase deficiency in malignant pleural mesotheliomas. Histopathol. 2012; 60(6B):E96-105.

22. Hylebos M, Van Camp G, van Meerbeeck JP, Op de Beeck K. The genetic landscape of malignant pleural mesothelioma: results from massively parallel sequencing. J Thorac Oncol. 2016;11(10):1615-26.

23. Widschwendter M, Fiegl H, Egle D, Mueller-Holzner E, Spizzo G, Marth C, et al. Epigenetic stem cell signature in cancer. Nat Genet. 2007;39(2):157-8

\section{Ready to submit your research? Choose BMC and benefit from:}

- fast, convenient online submission

- thorough peer review by experienced researchers in your field

- rapid publication on acceptance

- support for research data, including large and complex data types

- gold Open Access which fosters wider collaboration and increased citations

- maximum visibility for your research: over $100 \mathrm{M}$ website views per year

At BMC, research is always in progress.

Learn more biomedcentral.com/submissions 